\title{
High Mortality Rate in Hemodialysis Patients Who Undergo Invasive Cardiovascular Procedures Related to Peripheral Artery Disease
} - Community-Based Observational Study in Kumamoto Prefecture -

\author{
Seiji Hokimoto, MD, PhD; Kenji Sakamoto, MD, PhD; Tomonori Akasaka, MD; \\ Koichi Kaikita, MD, PhD; Osamu Honda, MD, PhD; Masahiro Naruse, MD, PhD; \\ Hisao Ogawa, MD, PhD for the Multi-center Study of Hemodialysis Patients \\ Undergoing Invasive Cardiovascular Procedures Study Investigators
}

\begin{abstract}
Background: Cardiovascular disease is a major cause of mortality in hemodialysis patients. The aim was to assess the relationship of various invasive cardiovascular procedures (ICP) to clinical outcome in hemodialysis patients.

Methods and Results: A total of 5,813 patients at 76 facilities were on maintenance hemodialysis in Kumamoto Prefecture. Of these, 4,807 patients at 58 institutions were enrolled. Of 4,807 patients, 212 ICP (4.4\%) were performed for various cardiovascular diseases in 189 patients (3.9\%). ICP included $\mathrm{PCI}(\mathrm{n}=80)$, endovascular treatment $(n=59)$, radiofrequency catheter ablation $(n=8)$, implantation of permanent pacemaker $(n=15)$ and ICD $(n=5)$, thoracotomy for valvular diseases $(n=16)$, CABG $(n=14)$, bypass surgery for peripheral artery disease (PAD; $n=8)$, and artificial vessel replacement for aneurysm or aortic dissection $(n=7)$. The overall mortality rate was $10.1 \%(19 / 189$ patients). The mortality rate was highest in patients who underwent ICP for PAD, compared with other ICP (PAD, 18.2\%; non-PAD, 6.7\%, $\mathrm{P}=0.017$ ). Infection and PAD were significant predictors of mortality (infection: OR, 8.30; 95\% Cl: 1.29-65.13, P=0.027; PAD: OR, 3.76; 95\% Cl: 1.35-10.48, P=0.012). The presence of inflammation $/ \mathrm{mal}-$ nutrition factors was associated with high mortality (OR, 15.49; 95\% Cl: 3.22-74.12, $\mathrm{P}=0.0006)$.
\end{abstract}

Conclusions: In this community-based registry study of 4,807 hemodialysis patients, the mortality rate of PAD patients was high despite ICP. (Circ J 2015; 79: 1269-1276)

Key Words: Arteriosclerosis; Cardiovascular disease; Hemodialysis; Mortality; Peripheral artery disease

$\mathbf{C}$ ardiovascular disease is a major cause of death in hemodialysis patients. ${ }^{1-7}$ Mortality in patients on maintenance hemodialysis in Japan was $9.4 \%$, and death from heart failure and myocardial infarction accounted for $30 \%$ according to the official 2011 report of the Japanese Society for Dialysis Therapy. ${ }^{8}$ Furthermore, the standardized mortality ratio for all-cause mortality was 4.6-fold higher in the hemodialysis patients compared with the general population. ${ }^{8}$ Age-adjusted mortality differences for cardiovascular and non-cardiovascular disease were 33.1 and 30.0 per 1,000 person-years, respectively. ${ }^{9}$ Although there are several studies on the outcome of invasive cardiovascular procedures (ICP), such as percutaneous coronary intervention (PCI), coronary artery bypass graft surgery (CABG) and endovascular treatment (EVT) for patients on hemodialysis, ${ }^{9-14}$ large-scale clinical studies in the cardiovascular field tend to exclude (or include only a few) patients on hemodialysis. Moreover, discussion tends to be focused on 1 treatment among various ICP from the point of view of cardiologists. Thus, there is incomplete understanding of the extent of the problem in hemodialysis patients. Giving that all-cause mortality rate is not low in hemodialysis patients who are at high risk of cardiovascular disease, and that kidney transplantation is not widespread in Japan, it is imperative to clarify the prognosis of patients who

Received December 1, 2014; revised manuscript received January 17, 2015; accepted February 3, 2015; released online March 17, 2015 Time for primary review: 17 days

Department of Cardiovascular Medicine, Graduate School of Medical Sciences, Kumamoto University, Kumamoto (S.H., K.S., T.A., K.K., H.O.); Committee of Dialysis Facilities in Kumamoto, Kumamoto (O.H., M.N.), Japan

Clinical Trial Registration Information: This study was registered in the UMIN protocol registration system (http://www.umin.ac.jp/ctr/) with the identification number UMIN000014604.

Mailing address: Seiji Hokimoto, MD, Department of Cardiovascular Medicine, Graduate School of Medical Sciences, Kumamoto University, 1-1-1 Honjo, Chuo-ku, Kumamoto 860-8556, Japan. E-mail: shokimot@kumamoto-u.ac.jp

ISSN-1346-9843 doi:10.1253/circj.CJ-14-1308

All rights are reserved to the Japanese Circulation Society. For permissions, please e-mail: cj@j-circ.or.jp 
5,813 hemodialysis patients in

Kumamoto Prefecture

(Jan 2011-Dec 2011)

1,006 patients in 18 facilities

with reject

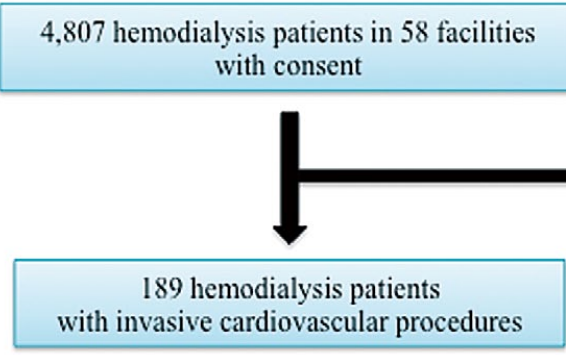

Figure 1. Subject selection. In Kumamoto Prefecture (1.8 million people), 5,813 patients were on hemodialysis in 76 facilities. Informed consent was obtained from 4,807 patients in 58 facilities, of the 5,813 hemodialysis patients. Of 4,807 patients, 189 patients underwent 212 invasive cardiovascular procedures (4.4\%) for various cardiovascular diseases.

undergo ICP. Cooperation between nephrologists and cardiologists to detect and take action against signs and symptoms of cardiovascular disease in hemodialysis patients in the early stage of the disease could potentially reduce the risk of cardiovascular events in these patients. ${ }^{15,16}$

The present study was designed to evaluate the incidence of ICP and ICP-related mortality in patients on maintenance hemodialysis in a community of 1.8 million people.

\section{Methods}

\section{Study Design and Participant Enrollment}

This study was a multicenter, prospective, registry study throughout Kumamoto Prefecture. Kumamoto Prefecture is located southwest of Tokyo, and has a population of approximately 1.8 million people. According to the 2011 official report of The Japanese Society for Dialysis Therapy, 5,813 patients at 76 facilities were on maintenance hemodialysis in Kumamoto Prefecture. Of these, 4,807 patients at 58 institutions were enrolled from January to December in 2011 in this study. Thus, the registration rate was $76.3 \%$ (58/76) for facilities, and $82.7 \%(4,807 / 5,813)$ for patients (Figure 1). We estimated all ICP that were performed for various cardiovascular diseases within 1 year (from January to December in 2012), and evaluated the follow-up data including mortality during a 24-month follow-up. We also examined anthropometric measures (eg, age, sex, weight, height), laboratory test results, and medical history (including pre-existing coronary event, stroke, peripheral vascular disease, and positive coronary angiography). Baseline clinical and laboratory data were obtained before hemodialysis, in patients who were stable, before undergoing ICP. At the time of ICP, patients with active infection or cancer were excluded. All data were collected and aggregated by a trained research team in the Division of Cardiovascular Disease at Kumamoto University.

\section{Definitions of ICP}

The ICP were as follows: PCI for coronary artery disease
(CAD), EVT for peripheral artery disease (PAD), radiofrequency catheter ablation (RFCA) for various arrhythmias, implantation of permanent pacemaker (PMI), implantation of implantable cardioverter defibrillator (ICD) or cardiac resynchronization therapy defibrillator (CRT-D), thoracotomy for various heart valve diseases, $\mathrm{CABG}$ for severe $\mathrm{CAD}$, bypass surgery for PAD, artificial vessel replacement for aneurysm or aortic dissection, and other procedures (eg, percutaneous transseptal mitral commissurotomy etc).

\section{Endpoint}

The study endpoint was all-cause mortality. All-cause death was classified into cardiac death, non-cardiac death, and sudden death. Cardiac death was defined as death from CAD, congestive heart failure, and fatal arrhythmia. The study was conducted in accordance with the Declaration of Helsinki and its amendments. The study protocol was in agreement with the guideline of the ethics committee of the institution and written informed consent was obtained from each patient or the family of the subject.

\section{Definition of Risk Factors}

Risk factors included hypertension, diabetes mellitus, and obesity. Hypertension was defined as systolic blood pressure $\geq 140 \mathrm{mmHg}$; diastolic blood pressure $\geq 90 \mathrm{mmHg}$; and/or the use of antihypertensive drugs. Diabetes mellitus was defined as fasting glucose $\geq 126 \mathrm{mg} / \mathrm{dl}$, 2-h blood glucose $\geq 200 \mathrm{mg} / \mathrm{dl}$, or use of a glucose-lowering drug or insulin. Body mass index (BMI) was calculated as body weight in kilograms divided by the height in meters squared $\left(\mathrm{kg} / \mathrm{m}^{2}\right)$.

\section{Statistical Analysis}

Continuous variables are expressed as mean \pm SD or median and interquartile, and differences between groups were evaluated using unpaired t-test or Mann-Whitney rank-sum test. Categorical variables are expressed as frequencies or percentages, and were compared using the Chi-squared test or Fisher exact test. Multiple logistic regression analysis was performed 


\begin{tabular}{lccc}
\hline \multicolumn{2}{l}{ Table 1. Baseline Clinical and Laboratory Data } & & \\
\\
$\mathrm{n}$ & ICP & No ICP & P-value \\
Age (years) & 189 & 4,618 & \\
Time on hemodialysis (years) & $69.5 \pm 11.0$ & $69.0 \pm 13.4$ & 0.681 \\
Height (cm) & $7.9 \pm 7.6$ & $8.7 \pm 8.0$ & 0.204 \\
Body weight before HD (kg) & $159.7 \pm 9.1$ & $158.1 \pm 10.0$ & 0.067 \\
BMl before HD (kg/m) & $57.8 \pm 10.1$ & $55.8 \pm 12.3$ & 0.051 \\
Male & $22.4 \pm 3.1$ & $22.2 \pm 4.6$ & 0.517 \\
Hypertension & 70.2 & 59.4 & 0.006 \\
Current smoking & 78.2 & 73.4 & 0.193 \\
Diabetes mellitus & 13.4 & 13.3 & 0.959 \\
BUN before HD (mg/dl) & 62.2 & 42.6 & $<0.001$ \\
Creatinine before HD (mg/dl) & $59.5 \pm 14.3$ & $60.5 \pm 15.5$ & 0.399 \\
Calcium (mg/dl) & $9.2 \pm 2.3$ & $9.7 \pm 2.8$ & 0.031 \\
Phosphorus (mg/dl) & $8.9 \pm 0.7$ & $9.0 \pm 0.8$ & 0.878 \\
Albumin (g/dl) & $5.0 \pm 1.4$ & $5.0 \pm 1.4$ & 0.811 \\
CRP (mg/dl) & $3.6 \pm 0.5$ & $3.6 \pm 0.4$ & 0.459 \\
Hemoglobin (g/dl) & $0.33(0.09-1.00)$ & $0.11(0.05-0.40)$ & $<0.001$ \\
Uric acid (mg/dl) & $10.3 \pm 1.2$ & $10.5 \pm 1.3$ & 0.183 \\
TC (mg/dl) & $7.8 \pm 6.2$ & $7.3 \pm 1.4$ & 0.002 \\
HDL-C (mg/dl) & $149.2 \pm 33.4$ & $154.7 \pm 35.8$ & 0.091 \\
Statin & $43.8 \pm 14.9$ & $45.9 \pm 14.6$ & 0.123 \\
Anti-hyperuricemia agent & 37.7 & 14.8 & $<0.001$ \\
Dataiven & 8.2 & 7.3 & 0.686 \\
\hline
\end{tabular}

Data given as mean \pm SD, \% or median (25-75\%). BMI, body mass index; BUN, blood urea nitrogen; CRP, C-reactive protein; HD, hemodialysis; HDL-C, high-density lipoprotein cholesterol; ICP, invasive cardiovascular procedure; TC, total cholesterol.

to determine the predictors for all-cause death in patients who underwent ICP. The results of multiple regression analysis are expressed as odds ratios (OR) for comparison of risk with $95 \%$ confidence intervals (CI). Predictor variables were included on the basis of theoretical grounds, clinical implications, and the results of bivariate analysis. For a better understanding of the relationship between ICP and mortality, we divided patients treated with various ICP into conventional and major categories of cardiovascular diseases, such as arrhythmia, CAD, PAD, and others, as model 1 . For model 2, mortality was classified into 2 categories: mortality related to PAD, and nonPAD. Moreover, because the number of events (all-cause death) was small, and to better distinguish patients with evidence of inflammation and/or malnutrition, a composite variable combining serum C-reactive protein (CRP), albumin, and creatinine, and body weight, was used to categorize the study population undergoing ICP into 3 subgroups according to the number of factors present (ie, no factor; multiple factors; and all factors). Low body weight was defined as BMI $<22 \mathrm{~kg} / \mathrm{m}^{2}$ (body weight varies with sex, and thus we used BMI as a marker of body weight), low serum creatinine was defined as $<8.4 \mathrm{mg} / \mathrm{dl}$ for women and $<9.4$ for men based on the median of the total group. Low serum albumin was defined as $<3.6 \mathrm{~g} / \mathrm{dl}$ (median). CRP $>0.1 \mathrm{mg} / \mathrm{dl}$ was defined as high. $\mathrm{P}<0.05$ indicated a statistically significant difference. SPSS ver. 22.0 (IBM Institute, Armonk, NY, USA) was used for all statistical analysis.

\section{Results}

\section{Patient Characteristics}

Table 1 lists the baseline characteristics of patients who underwent ICP (ICP arm, n=189) or not (no-ICP arm, n=4,618) during 2011. Age and duration of hemodialysis were similar between the 2 groups. The prevalences of male sex and diabetes mellitus were higher in the ICP arm than in the no-ICP arm (ICP vs. no ICP: male, $70.2 \%$ vs. $59.4 \%, \mathrm{P}=0.006$; diabetes mellitus, $62.2 \%$ vs. $42.6 \%, \mathrm{P}<0.001)$. There were significant differences in serum creatinine, serum CRP, and serum uric acid between the ICP arm and no-ICP arm (ICP vs. no ICP: creatinine, $9.2 \pm 2.3 \mathrm{mg} / \mathrm{dl}$ vs. $9.7 \pm 2.8 \mathrm{mg} / \mathrm{dl}, \mathrm{P}=0.031$; CRP, $1.46 \pm 3.03 \mathrm{mg} / \mathrm{dl}$ vs. $0.63 \pm 1.93 \mathrm{mg} / \mathrm{dl}, \mathrm{P}<0.0001$; uric acid, $7.8 \pm 6.2 \mathrm{mg} / \mathrm{dl}$ vs. $7.3 \pm 1.4 \mathrm{mg} / \mathrm{dl}, \mathrm{P}=0.002)$. There were no patients with missing data.

\section{Details of ICP}

Table 2 lists the ICP patient details. The total number of ICP conducted in 2011 was 212. This included PCI in 80 cases, EVT in 59, RFCA in 8, PMI in 15, ICD in 5, surgical valvular repair in $16, \mathrm{CABG}$ in 14 , bypass operation for PAD in 8 , and artificial vessel replacement in 7 (Table 2). The number of patients who underwent a single ICP was 169 while 20 underwent more than $1 \mathrm{ICP}$. The latter group included 9 patients who underwent both PCI and EVT (one of them also underwent $\mathrm{CABG}$ ), 3 patients received PCI and procedures for arrhythmia (2 RFCA and 1 ICD), and 2 patients underwent both EVT and bypass surgery for PAD. Of the 6 patients with thoracotomy for valve disease, 2 received CABG, 1 received PMI, 1 underwent EVT, and another patient received ICD.

All-cause mortality based on ICP was $10.4 \%$. The mortality rate was significantly higher in patients who underwent CABG for CAD and bypass surgery or EVT for PAD, compared with other ICP (CABG, 14.3\%; bypass surgery for PAD, 37.5\%; EVT for PAD, $13.6 \%$; Table 2 ). In contrast, the mortality rate of patients with arrhythmia-related ICP, such as RFCA and ICD, was relatively low (RFCA, 0\%; PMI, 13.3\%; ICD, 0\%). 


\begin{tabular}{|c|c|c|c|c|c|c|}
\hline \multirow{2}{*}{ ICP } & \multirow{2}{*}{$\mathbf{n}$} & \multirow{2}{*}{$\begin{array}{l}\text { All-cause } \\
\text { death (n) }\end{array}$} & \multicolumn{4}{|c|}{ Reason for death } \\
\hline & & & Cardiac & Sudden & Non-cardiac & Unknown \\
\hline \multicolumn{7}{|l|}{ Single ICP } \\
\hline $\mathrm{PCl}$ & 80 & 6 & 1 & 2 & 3 & 0 \\
\hline EVT for PAD & 59 & 8 & 0 & 2 & 6 & 0 \\
\hline RFCA & 8 & 0 & 0 & 0 & 0 & 0 \\
\hline PMI & 15 & 2 & 0 & 0 & 1 & 1 \\
\hline ICD & 5 & 0 & 0 & 0 & 0 & 0 \\
\hline CRT-D & 0 & 0 & 0 & 0 & 0 & 0 \\
\hline Surgery for valve disease & 16 & 1 & 1 & 0 & 0 & 0 \\
\hline CABG & 14 & 2 & 0 & 0 & 2 & 0 \\
\hline Surgery for $P A D$ & 8 & 3 & 1 & 0 & 2 & 0 \\
\hline Vessel replacement for $A A / A D$ & 7 & 0 & 0 & 0 & 0 & 0 \\
\hline Total & 212 & 22 & 3 & 4 & 14 & 1 \\
\hline \multicolumn{7}{|l|}{ Mortality vs. No. of ICP } \\
\hline 1 & 169 & 17 & & & & \\
\hline 2 & 17 & 1 & & & & \\
\hline 3 & 3 & 1 & & & & \\
\hline
\end{tabular}

\begin{tabular}{|c|c|c|c|c|c|c|c|c|}
\hline \multirow{2}{*}{ ICP } & \multirow{2}{*}{$\begin{array}{c}\text { Mortality } \\
(\%)\end{array}$} & \multirow{2}{*}{ P-value } & \multicolumn{5}{|c|}{ Clinical events during follow-up } & \multirow{2}{*}{$\begin{array}{l}\text { Total } \\
\text { events }\end{array}$} \\
\hline & & & MI & CHF & Arrhythmia & Malignancy & Infection & \\
\hline \multicolumn{9}{|l|}{ Single ICP } \\
\hline $\mathrm{PCl}$ & 7.5 & & 1 & 6 & 3 & 1 & 4 & 21 \\
\hline EVT for PAD & 13.6 & & 0 & 1 & 2 & 0 & 2 & 13 \\
\hline RFCA & 0 & & 0 & 0 & 0 & 0 & 0 & 0 \\
\hline PMI & 13.3 & & 0 & 0 & 0 & 2 & 0 & 4 \\
\hline ICD & 0 & & 0 & 0 & 0 & 1 & 0 & 1 \\
\hline CRT-D & 0 & & 0 & 0 & 0 & 0 & 0 & 0 \\
\hline Surgery for valve disease & 6.25 & & 0 & 0 & 0 & 0 & 0 & 1 \\
\hline CABG & 14.3 & & 1 & 0 & 0 & 0 & 0 & 3 \\
\hline Surgery for PAD & 37.5 & & 1 & 1 & 0 & 0 & 1 & 6 \\
\hline Vessel replacement for AA/AD & 0 & & 0 & 0 & 1 & 0 & 0 & 1 \\
\hline Total & 10.4 & & 3 & 8 & 6 & 4 & 7 & 50 \\
\hline \multicolumn{9}{|l|}{ Mortality vs. No. of ICP } \\
\hline 1 & 10.1 & & & & & & & \\
\hline 2 & 5.9 & 0.3475 & & & & & & \\
\hline 3 & 33.3 & & & & & & & \\
\hline
\end{tabular}

$\mathrm{AA}$, aortic aneurysm; $\mathrm{AD}$, aortic dissection; $\mathrm{CABG}$, coronary artery bypass graft; $\mathrm{CHF}$, congestive heart failure; CRT-D, cardiac resynchronization therapy defibrillator; EVT, endovascular treatment; ICD, implantable cardioverter defibrillator; MI, myocardial infarction; PAD, peripheral arterial disease; $\mathrm{PCl}$, percutaneous coronary intervention; PMI, implantation of permanent pacemaker; RFCA, radiofrequency catheter ablation. Other abbreviation as in Table 1.

\begin{tabular}{|lcccc|}
\hline $\begin{array}{l}\text { Table 3. Mortality vs. CV Disease } \\
\text { Category }\end{array}$ & $\mathbf{n}$ & mortality & $\%$ & P-value \\
Model 1 & & & & \\
Arrhythmia & 26 & 2 & 7.69 & \\
Coronary artery disease & 90 & 6 & 6.67 & 0.1259 \\
PAD & 55 & 10 & 18.18 & \\
Others (valvular+aortic disease) & 18 & 1 & 5.56 & \\
Model 2 & & & & \\
PAD & 55 & 10 & 18.18 & \\
Non-PAD & 134 & 9 & 6.72 & 0.0173 \\
Total & 189 & 19 & 10.05 & \\
\hline
\end{tabular}

Treatments for valvular disease and aortic aneurysm/dissection were categorized as others. CV, cardiovascular. Other abbreviation as in Table 2. 
Table 4. Predictors of Mortality According to Disease

\begin{tabular}{|c|c|c|c|c|c|c|}
\hline \multirow{2}{*}{ Variable } & \multicolumn{3}{|c|}{ Univariate Analysis } & \multicolumn{3}{|c|}{ Multivariate Analysis } \\
\hline & OR & $(95 \% \mathrm{Cl})$ & P-value & OR & $(95 \% \mathrm{Cl})$ & P-value \\
\hline $\mathrm{CHF}$ & 1.294 & $(0.150-11.120)$ & 0.815 & 1.222 & $(0.125-11.951)$ & 0.863 \\
\hline Malignancy & 3.093 & $(0.305-31.313)$ & 0.339 & 5.724 & $(0.496-66.014)$ & 0.162 \\
\hline Infection & 6.549 & $(1.022-41.970)$ & 0.047 & 9.160 & (1.288-65.133) & 0.027 \\
\hline PAD & 3.086 & $(1.178-8.085)$ & 0.022 & 3.755 & $(1.346-10.476)$ & 0.012 \\
\hline
\end{tabular}

$\mathrm{Cl}$, confidence interval; OR, odds ratio. Other abbreviations as in Table 2.

\begin{tabular}{|c|c|c|c|}
\hline & Death & Survival & P-value \\
\hline $\mathrm{n}$ & 19 & 170 & \\
\hline Age (years) & $73.6 \pm 10.3$ & $69.2 \pm 11.0$ & 0.127 \\
\hline Time on dialysis (years) & $11.1 \pm 10.8$ & $7.5 \pm 7.2$ & 0.095 \\
\hline Height (cm) & $156.6 \pm 11.3$ & $160.0 \pm 8.8$ & 0.194 \\
\hline Body weight before HD (kg) & $51.9 \pm 9.5$ & $58.3 \pm 10.0$ & 0.035 \\
\hline BMI before HD $\left(\mathrm{kg} / \mathrm{m}^{2}\right)$ & $21.0 \pm 3.4$ & $22.5 \pm 3.0$ & 0.106 \\
\hline Male & 68.4 & 70.0 & 0.887 \\
\hline Hypertension & 53.8 & 80.6 & 0.026 \\
\hline Current smoking & 8.3 & 13.4 & 0.672 \\
\hline Diabetes & 53.8 & 63.0 & 0.517 \\
\hline Statin & 46.2 & 36.8 & 0.508 \\
\hline BUN before HD (mg/dl) & $55.6 \pm 14.1$ & $59.8 \pm 14.3$ & 0.306 \\
\hline Creatinine before $\mathrm{HD}(\mathrm{mg} / \mathrm{dl})$ & $7.7 \pm 1.2$ & $9.3 \pm 2.3$ & 0.012 \\
\hline$\Delta \mathrm{Cr}(\mathrm{mg} / \mathrm{dl})$ & $5.0 \pm 1.1$ & $6.1 \pm 1.5$ & 0.013 \\
\hline $\mathrm{Ca}(\mathrm{mg} / \mathrm{dl})$ & $9.1 \pm 1.0$ & $8.9 \pm 0.7$ & 0.310 \\
\hline Phosphorus (mg/dl) & $4.5 \pm 1.2$ & $5.1 \pm 1.4$ & 0.179 \\
\hline Albumin (g/dl) & $3.4 \pm 0.5$ & $3.6 \pm 0.4$ & 0.040 \\
\hline $\operatorname{CRP}(\mathrm{mg} / \mathrm{dl})$ & $2.07(0.12-2.70)$ & $0.31(0.08-0.86)$ & 0.047 \\
\hline Hemoglobin (g/dl) & $10.2 \pm 1.7$ & $10.3 \pm 1.1$ & 0.693 \\
\hline Uric acid (mg/dl) & $7.2 \pm 1.0$ & $7.8 \pm 6.4$ & 0.756 \\
\hline $\mathrm{TC}(\mathrm{mg} / \mathrm{dl})$ & $148.3 \pm 36.8$ & $149.3 \pm 33.3$ & 0.920 \\
\hline $\mathrm{HDL}-\mathrm{C}(\mathrm{mg} / \mathrm{dl})$ & $40.4 \pm 12.5$ & $44.0 \pm 15.1$ & 0.488 \\
\hline Statin & 46.2 & 36.8 & 0.508 \\
\hline Anti-hyperuricemia agent & 7.7 & 8.2 & 0.948 \\
\hline
\end{tabular}

Data given as mean \pm SD, \% or median (25-75\%). Abbreviations as in Table 1.

The non-cardiac death rate $(14 / 22 ; 63.6 \%)$ was higher than that of cardiac death $(3 / 22 ; 13.6 \%)$ according to ICP basement $(\mathrm{P}<0.001)$. This indicates that all-cause mortality was high despite the success of ICP, and thus we need to take into account the general condition of hemodialysis patients who undergo ICP. Although the number of ICP did not correlate with mortality, the mortality rate tended to be higher in patients who underwent $3 \mathrm{ICP}$ than 1 or $2 \mathrm{ICP}$, albeit statistically insignificantly (mortality: $10.1 \%, 5.9 \%, 33.3 \%$ for 1,2 , and 3 procedures, respectively, $\mathrm{P}=0.347$; Table 2 ). The mean days from ICP to death was 393 days.

\section{High PAD-Related Mortality}

We also evaluated mortality according to conventional and major categories of cardiovascular disease (Table 3). Among the 4 major categories (arrhythmia, CAD, PAD, and others [valvular or aortic disease], model 1), mortality related to PAD was the highest (PAD, 18.2\%; arrhythmia, 7.7\%; CAD, $6.7 \%$; others, 5.3\%; $\mathrm{P}=0.126)$. Based on this finding, we divided the patients into the PAD-related mortality group and
non-PAD-related mortality group (model 2). There was a significant difference in the mortality rate between the 2 groups $(\mathrm{P}=0.017)$.

\section{Predictors of Mortality According to Disease}

Multivariate logistic regression analysis for mortality according to the various diseases such as congestive heart failure, malignancy, infection, and PAD, was performed to examine the significant determinants of death after ICP in hemodialysis patients (Table 4). The selection of congestive heart failure, malignancy, and infection in the aforementioned analysis was based on their association with mortality in hemodialysis patients. ${ }^{8}$ The presence of infection or PAD in hemodialysis patients treated with ICP was a significant predictor of mortality (infection: OR, 9.16; 95\% CI: 1.29-65.13, P=0.027; PAD: OR, 3.76; 95\% CI: 1.35-10.48, P=0.012; Table 4).

\section{Laboratory Data}

We compared the clinical features and results of laboratory tests with regard to death (Table 5). Among the clinical fea- 


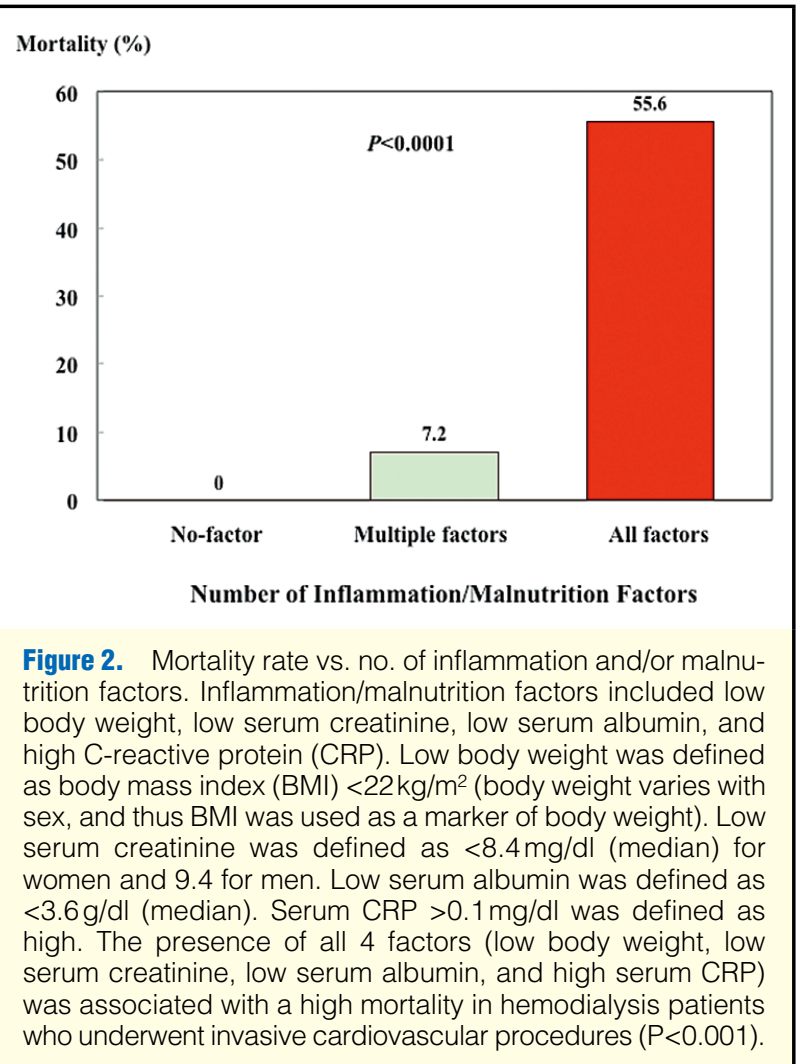

tures examined, body weight and the prevalence of hypertension were significantly lower in the death group. Furthermore, serum creatinine and albumin were lower in the death group than the survivor group. High serum CRP was found in the death group compared with the survivor group. In contrast, the lipid profile was similar between the 2 groups. Four factors, including body weight, serum creatinine, serum albumin, and serum CRP are conventional risk factor for prognosis in hemodialysis patients, and they are considered as inflammation/malnutrition factors. ${ }^{17}$ The presence of all 4 factors (ie, low body weight, low serum creatinine and albumin, and high serum CRP) was associated with a high mortality in hemodialysis patients who underwent ICP $(\mathrm{P}<0.001$; Figure 2). Moreover, there were differences in hypertension, inflammation/malnutrition factors including body weight, serum creatinine, serum albumin, and serum CRP between the death and survivor patients undergoing ICP. Age is also generally considered as an important factor for mortality. Therefore, in this study, we selected the factors of inflammation/malnutrition, hypertension, and age on multiple regression analysis. The inflammation/malnutrition factor was a significant predictor for mortality among age, hypertension, and inflammation/malnu- trition factors (OR, 15.44; 95\% CI: 3.22-74.12, $\mathrm{P}=0.0006$; Table 6).

\section{Discussion}

It is important to assess the current status of cardiovascular diseases in hemodialysis patients in the community at large, given that cardiovascular diseases are the major cause of death in these patients. ${ }^{8,18-22}$ In this study, we assessed the incidence and mortality rate related to ICP in hemodialysis patients in a community of 1.8 million people. The major findings of the present study were as follows: (1) the incidence of ICP in hemodialysis patients living in the community was 3.9\% (189/4,807 patients); (2) the mortality rate in patients who underwent ICP was $10.1 \%$ (19/189 patients) within a 1-year follow-up period; (3) the mortality rate among hemodialysis patients who underwent ICP was highest in patients who received EVT or bypass surgery for PAD; (4) systemic infection and PAD were significant predictors of mortality in hemodialysis patients treated with ICP; (5) the rate of non-cardiac death was higher than cardiac death in hemodialysis patients despite the success of ICP; and (6) inflammation/malnutrition factors increased the mortality rate, similar to the results of previous studies. ${ }^{17,23}$

All-cause mortality of patients who underwent EVT or bypass surgery for PAD was the highest among those who underwent ICP. These results are in agreement with previous reports that showed poor prognosis in PAD patients irrespective of hemodialysis. ${ }^{24-26}$ The cause of death in patients who underwent ICP for PAD was not necessarily cardiac death alone, and patients with PAD may have polyvascular atherosclerotic disease, with associated comorbidity of CAD such as myocardial infarction, or cerebrovascular diseases such as stroke, or transient ischemic attack. ${ }^{27,28}$ PAD is reported to progress in patients with renal dysfunction and to become the end stage of various atherosclerotic diseases, especially in hemodialysis patients, and to be associated with a high incidence of serious complications such as limb ischemia and infection. ${ }^{15,24,29,30}$ In short, the prognosis of such patients may be unfavorable in general. This could explain the high mortality rate in hemodialysis patients who underwent ICP for PAD.

Hemodialysis patients referred from private hemodialysis clinics to hospitals with cardiovascular facilities must undergo detailed medical examination before the development of serious and severe comorbidity such as cardiovascular disease, especially atherosclerotic disease..$^{15,16}$ Cooperation between nephrologists and cardiologists is important in the management of such patients for early identification of cardiovascular disease in hemodialysis patients, and to detect and respond to early clinical signs and symptoms of cardiovascular disease, as well as to improve the prognosis of patients on maintenance hemodialysis.

The mortality rate in hemodialysis patients is reported to be low in Japan compared with the USA and Europe. ${ }^{31}$ PAD is associated with increased risk of cardiovascular mortality in

\begin{tabular}{|c|c|c|c|c|c|c|}
\hline \multirow{2}{*}{ Variable } & \multicolumn{3}{|c|}{ Univariate Analysis } & \multicolumn{3}{|c|}{ Multivariate Analysis } \\
\hline & OR & $(95 \% \mathrm{Cl})$ & P-value & OR & $(95 \% \mathrm{Cl})$ & P-value \\
\hline Age & 1.040 & (0.989-1.095) & 0.129 & 1.003 & $(0.941-1.069)$ & 0.936 \\
\hline Hypertension & 0.281 & $(0.087-0.906)$ & 0.034 & 0.429 & $(0.109-1.694)$ & 0.227 \\
\hline Inflammation/malnutrition & 21.250 & $(4.761-94.840)$ & $<0.0001$ & 15.449 & $(3.220-74.124)$ & 0.0006 \\
\hline
\end{tabular}

Abbreviations as in Table 4. 
many developed countries. Although Japanese patients have significantly better prognosis compared with the other countries, there may be a possibility that early care for hemodialysis patients with PAD would result in reduced mortality. ${ }^{32}$ Even in Japan, however, mortality in hemodialysis patients who undergo ICP, especially those with atherosclerotic disease, is not low. ${ }^{8}$ One reason for this is the generally poor general condition of hemodialysis patients compared with non-hemodialysis patients. ${ }^{17,23}$ In the present study, in addition to the risk factors of PAD and infection, inflammation and/or malnutrition factors, including low serum creatinine and albumin, high serum CRP, and low body weight, were associated with high mortality, as reported previously. ${ }^{17,23}$ In addition to successful ICP, the attending physicians should aim to improve general patient status, and the laboratory data, in order to reduce the mortality rate in hemodialysis patients.

In the present study, the mortality rate was low in patients who underwent arrhythmia-related ICP, such as RFCA, ICD, and PMI, compared with other ICP $(2 / 28$ patients, $7.1 \%) .{ }^{33} \mathrm{In}$ particular, no deaths were registered in patients who received ICD implantation for fatal arrhythmia. While the exact reason for the low mortality is unknown, it could be related to the attending physicians themselves (strict management of RFCA, PMI and ICD), or to the hemodialysis patients themselves (preferral of medical therapy over invasive treatment). Further studies are needed to explore this issue in more detail.

\section{Clinical Implications}

The all-cause mortality of patients who received ICP for PAD was higher than that unrelated to PAD despite the low rate of cardiac death. It is possible that these patients suffered from advanced and progressive PAD due to late diagnosis. Early detection and management of cardiovascular disease, especially PAD, are important, and, together with cooperation between nephrologists and cardiologists, may improve the clinical outcome of hemodialysis patients who undergo ICP.

\section{Study Limitations}

It is possible that this study did not include patients with PAD who were not suitable for EVT or bypass surgery due to extremely poor general condition or advanced state of PAD. This may have caused underestimation of the present mortality rate. The number of deaths was low, but the aim of the study was to describe the current status of hemodialysis patients undergoing ICP for various cardiovascular diseases in an administrative unit of local government with a population of 1.8 million. From this observational study, the high mortality in hemodialysis patients with PAD despite ICP emphasizes the need for cooperation between nephrologists and cardiologists in the management of hemodialysis patients. According to the rate of death associated with infection, the present study population was similar to that in the previous study. In this study, patients with active infection or cancer were excluded at the time of enrollment. In the follow-up period, some infections requiring hospitalization developed, such as pneumonia, sepsis, bacteremia, infection of urinary tract, hepatitis, and infection of cutaneous ulcer, but infection was not classified into specific disease.

\section{Conclusions}

This community-based registry study was conducted in patients on maintenance hemodialysis. Of 4,807 hemodialysis patients, the mortality rate of patients with PAD was high despite ICP.

\section{Acknowledgments}

We thank the medical secretaries, Aya Miyazaki, Akiyo Kikuchi, Kyoko Watanabe, Hiroko Koga, Yurie Maeda, Chihiro Yamamoto and Rina Usui at Kumamoto University Hospital for collecting the data.

\section{Funding Sources}

This study was supported in part by grants-in-aid for scientific research from the Ministry of Education, Culture, Sports, Science and Technology, Japan.

\section{Disclosures}

All authors declare no conflicts of interest.

\section{References}

1. Levey AS, Beto JA, Coronado BE, Eknoyan G, Foley RN, Kasiske $\mathrm{BL}$, et al. Controlling the epidemic of cardiovascular disease in chronic renal disease: What do we know? What do we need to learn? Where do we go from here? National Kidney Foundation Task Force on Cardiovascular Disease. Am J Kidney Dis 1998; 32: 853-906.

2. Shlipak MG, Simon JA, Grady D, Lin F, Wenger NK, Furberg CD, et al. Renal insufficiency and cardiovascular events in postmenopausal women with coronary heart disease. J Am Coll Cardiol 2001; 38: $705-711$.

3. Foley RN, Parfrey PS, Sarnak MJ. Epidemiology of cardiovascular disease in chronic renal disease. J Am Soc Nephrol 1998; 9: S16S23.

4. Sarnak MJ, Levey AS, Schoolwerth AC, Coresh J, Culleton B, Hamm LL, et al. Kidney disease as a risk factor for development of cardiovascular disease: A statement from the American Heart Association Councils on Kidney in Cardiovascular Disease, High Blood Pressure Research, Clinical Cardiology, and Epidemiology and Prevention. Circulation 2003; 108: 2154-2169.

5. Stack AG, Bloembergen WE. Prevalence and clinical correlates of coronary artery disease among new dialysis patients in the United States: A cross-sectional study. J Am Soc Nephrol 2001; 12: 15161523.

6. Sato A. Predicting cardiac and all-cause death in asymptomatic patients on hemodialysis: Importance of training in interpretation of $\beta$-Methyl Iodophenyl-pentadecanoic Acid Single-Photon Emission Computed Tomography (BMIPP SPECT) imaging. Circ J 2015; 79: $47-48$.

7. Kiriyama T, Kumita SI, Moroi M, Nishimura T, Tamaki N, Hasebe $\mathrm{N}$, et al. Interpretative variability and its impact on the prognostic value of myocardial fatty acid imaging in asymptomatic hemodialysis patients in a multicenter trial in Japan. Circ J 2015; 79: 153-160.

8. Nakai S, Watanabe Y, Masakane I, Wada A, Shoji T, Hasegawa T, et al. Overview of regular dialysis treatment in Japan (as of 31 December 2011). Ther Apher Dial 2013; 17: 567-611.

9. Wakasugi M, Kazama JJ, Yamamoto S, Kawamura K, Narita I. Cause-specific excess mortality among dialysis patients: Comparison with the general population in Japan. Ther Apher Dial 2013; 17: 298-304.

10. Charytan D, Mauri L, Agarwal A, Servoss S, Scirica B, Kuntz RE. The use of invasive cardiac procedures after acute myocardial infarction in long-term dialysis patients. Am Heart J 2006; 152: 558-564.

11. Hemmelgarn BR, Southern D, Culleton BF, Mitchell LB, Knudtson ML, Ghali WA, et al. Survival after coronary revascularization among patients with kidney disease. Circulation 2004; 110: 1890-1895.

12. Kumada Y, Aoyama T, Ishii H, Tanaka M, Kawamura Y, Takahashi $\mathrm{H}$, et al. Long-term outcome of percutaneous transluminal angioplasty in chronic haemodialysis patients with peripheral arterial disease. Nephrol Dial Transplant 2008; 23: 3996-4001.

13. O'Hare AM, Sidawy AN, Feinglass J, Merine KM, Daley J, Khuri $\mathrm{S}$, et al. Influence of renal insufficiency on limb loss and mortality after initial lower extremity surgical revascularization. J Vasc Surg 2004; 39: 709-716.

14. Ishii H, Kumada Y, Toriyama T, Aoyama T, Takahashi H, Murohara T. Prognostic values of C-reactive protein levels on clinical outcome after endovascular therapy in hemodialysis patients with peripheral artery disease. J Vasc Surg 2010; 52: 854-859.

15. Belch JJ, Topol EJ, Agnelli G, Bertrand M, Califf RM, Clement DL, et al. Critical issues in peripheral arterial disease detection and management: A call to action. Arch Intern Med 2003; 163: 884-892.

16. DeLoach SS, Mohler ER 3rd. Peripheral arterial disease: A guide for nephrologists. Clin J Am Soc Nephrol 2007; 2: 839-846.

17. Liu Y, Coresh J, Eustace JA, Longenecker JC, Jaar B, Fink NE, et 
al. Association between cholesterol level and mortality in dialysis patients: Role of inflammation and malnutrition. JAMA 2004; 291: 451-459.

18. Martin D, Wallace D, Crowe M, Rush C, Tosenovsky P, Golledge J. Association of total white cell count with mortality and major adverse events in patients with peripheral arterial disease: A systematic review. Eur J Vasc Endovasc Surg 2014; 47: 422-432.

19. Go AS, Chertow GM, Fan D, McCulloch CE, Hsu CY. Chronic kidney disease and the risks of death, cardiovascular events, and hospitalization. N Engl J Med 2004; 351: 1296-1305.

20. Bansal N, Hsu CY, Chandra M, Iribarren C, Fortmann SP, Hlatky $\mathrm{MA}$, et al. Potential role of differential medication use in explaining excess risk of cardiovascular events and death associated with chronic kidney disease: A cohort study. BMC Nephrol 2011; 12: 44.

21. Bonello L, De Labriolle A, Roy P, Steinberg DH, Okabe T, Pinto Slottow TL, et al. Impact of optimal medical therapy and revascularization on outcome of patients with chronic kidney disease and on dialysis who presented with acute coronary syndrome. Am J Cardiol 2008; 102: 535-540.

22. Herzog CA, Asinger RW, Berger AK, Charytan DM, Diez J, Hart $\mathrm{RG}$, et al. Cardiovascular disease in chronic kidney disease. A clinical update from Kidney Disease: Improving Global Outcomes (KDIGO). Kidney Int 2011; 80: 572-586.

23. Pifer TB, McCullough KP, Port FK, Goodkin DA, Maroni BJ, Held $\mathrm{PJ}$, et al. Mortality risk in hemodialysis patients and changes in nutritional indicators: DOPPS. Kidney Int 2002; 62: 2238-2245.

24. O'Hare AM, Bertenthal D, Shlipak MG, Sen S, Chren MM. Impact of renal insufficiency on mortality in advanced lower extremity peripheral arterial disease. J Am Soc Nephrol 2005; 16: 514-519.

25. Malyar N, Furstenberg T, Wellmann J, Meyborg M, Luders F, Gebauer K, et al. Recent trends in morbidity and in-hospital outcomes of in-patients with peripheral arterial disease: A nationwide population-based analysis. Eur Heart J 2013; 34: 2706-2714.

26. Mueller T, Hinterreiter F, Luft C, Poelz W, Haltmayer M, Dieplinger B. Mortality rates and mortality predictors in patients with symptomatic peripheral artery disease stratified according to age and diabetes. J Vasc Surg 2014; 59: 1291-1299.

27. Bhatt DL, Steg PG, Ohman EM, Hirsch AT, Ikeda Y, Mas JL, et al International prevalence, recognition, and treatment of cardiovascular risk factors in outpatients with atherothrombosis. JAMA 2006; 295: $180-189$.

28. Steg PG, Bhatt DL, Wilson PW, D'Agostino R Sr, Ohman EM, Rother J, et al. One-year cardiovascular event rates in outpatients with atherothrombosis. JAMA 2007; 297: 1197-1206.

29. Barrett BJ, Parfrey PS, Morgan J, Barre P, Fine A, Goldstein MB, et al. Prediction of early death in end-stage renal disease patients starting dialysis. Am J Kidney Dis 1997; 29: 214-222.

30. Koch M, Trapp R, Kulas W, Grabensee B. Critical limb ischaemia as a main cause of death in patients with end-stage renal disease: A single-centre study. Nephrol Dial Transplant 2004; 19: 2547-2552.

31. Kuhlmann MK, Yoshino M, Levin NW. Differences in cardiovascular mortality rates among hemodialysis patients in the United States and Japan: The importance of background cardiovascular mortality. Hemodial Int 2004; 8: 394-399.

32. Rajagopalan S, Dellegrottaglie S, Furniss AL, Gillespie BW,
Satayathum S, Lameire N, et al. Peripheral arterial disease in patients with end-stage renal disease: Observations from the Dialysis Outcomes and Practice Patterns Study (DOPPS). Circulation 2006; 114: $1914-1922$.

33. Cuculich PS, Sanchez JM, Kerzner R, Greenberg SL, Sengupta J, Chen J, et al. Poor prognosis for patients with chronic kidney disease despite ICD therapy for the primary prevention of sudden death. Pacing Clin Electrophysiol 2007; 30: 207-213.

\section{Appendix}

\section{Investigators}

Kenji Arizono, Kumamoto Chuo Hospital Nephrology; Syouji Fujisawa, Tamana Urological Clinic; Hiroyoshi Fukui, Chuojin Clinic; Jun Fukushima, Fukushima Clinic; Michiaki Hara, Hara Internal Medicine Clinic; Sadanobu Higuchi, Kamiamakusa General Hospital; Osamu Honda, Kengun Clinic; Nobuhiko Ikezaki, Jinseikai Clinic Ozu; Sadayoshi Ikezaki, Otemachi Clinic; Masayuki Imafuji, Ueki Imafuji Clinic; Takashi Ishimatsu, Hirayama Urology Department Clinic; Hitoshi Iwashita, Tamana Central Hospital; Yuichi Iwashita, Tsutsumi Hospital; Yukitsugu Kawabata, Tsuruta Hospital; Masaharu Kawatomi, Sakura Hospital; Syunichi Kimura, Shimada Hospital; Mitsuhiro Kodama, Taragi Municipal Hospital; Atsuko Kugiyama, Uto Chuo Clinic; Kuniharu Kuwahara, Kuwahara Clinic; Kazuaki Mabe, Mabe Hospital; Jiro Machida, Saiseikai Kumamoto Hospital Kidney Urology Center; Akihito Maehara, Maehara Urology Department Clinic; Yoshinari Matsunaga, Amakusa Regional Medical Center; Noboru Matsuoka, Matsuoka Internal Medicine Clinic; Kazunori Matsushita, Kazutaka Matsushita, Akebono Clinic; Atsushi Migita, Migita Clinic; Tetsuaki Miyamoto, Mashiki Chuo Hospital; Takashi Miyanaka, Kumamoto City Hospital Nephrology; Kensuke Mizutari, Kumamoto Shinto General Hospital; Yasuteru Miyamoto, Miyamoto Internal Medicine Clinic; Yasufumi Nabekura, Kumamoto Urological Hospital; Takeshi Motoyama, Konan Hospital; Hiroto Nagano, Amakusa First Hospital; Minoru Nagayoshi, Nagayoshi Clinic; Takamichi Nakamura, Nakamura Internal Medicine Clinic; Takehiko Nakano, Nakano Clinic; Kiyoshi Nakashita, Internal Medicine Kumamoto Clinic; Masahiro Naruse, Tamana Daiichi Clinic; Akihiro Nojiri, Kumamoto Urological Hospital; Tetsuya Oda, Midorikawa Clinic; Yoshiaki Otsuka, Otsuka Clinic; Shunichi Sakaguchi, Midorigaoka Clinic; Toshihiko Sakanashi, Sakanashi Heart Clinic; Yoko Seto, Seto Hospital; Hidetaka Shimada, Shimada Hospital; Takafumi Shimomura, Aso-Onsen Hospital; Munemasa Tajiri, Jinseikai Clinic Kurokami; Tetsuya Tajiri, Jinseikai Clinic Nagamine; Tomi Takamiya, Takamiya Clinic; Michiyo Takeshita, Amakusa Chuo General Hospital; Hiroshi Terasaki, Terasaki Clinic; Takeshi Tsuru, Oyano Clinic; Kazuma Tsuruta, Kashima Clinic; Syoichiro Tsuzaki, Hirayama Clinic; Masatoshi Tsukamoto, Arao Central Hospital; Souichi Uekihara, Japanese Red Cross Kumamoto Hospital Nephrology; Saishi Uemura, Uemura Cardiology Clinic; Hideaki Uchigashima, Konan Hospital; Shinichi Uemura, Aso Tateno Hospital; Kunio Yamada, Arao Clinic; Shinjiro Yano, Kyushu Memorial Hospital; Naruhiro Yasumoto, Yasumoto Internal Medicine Clinic; Noburo Tazoe, Kazuhiko Nishi, Committee of Dialysis Facilities in Kumamoto; Seijji Hokimoto, Kenji Sakamoto, Tomonori Akasaka, Koichi Kaikita, Hisao Ogawa, Kumamoto University. 